\title{
Modified DOA Estimation with an Unfolded Co-prime Linear Array
}

\author{
Xiao Yang, Yide Wang, and Pascal Chargé
}

\begin{abstract}
Direction-of-arrival estimation with unfolded coprime linear arrays has been investigated a lot thanks to the large aperture, excellent performance and full degrees-of-freedom (DOF). Besides, it has been admitted that the ambiguity problem can be suppressed thanks to the co-prime property. However, it is not always true. When there are two different DOAs having the same directional vectors with a given DOA for the two subarrays respectively, ambiguities would still be found at the angles of which the corresponding directional vectors can be represented as linear combinations of the directional vectors of true DOAs. Surprisingly, this problem has been ignored by all the existing work. In this letter, this ambiguity problem is discussed for the first time to our best knowledge, and an appropriate method for fixing it is proposed. Compared to the original method, the proposed method contains all the advantages of unfolded co-prime linear arrays, and can solve the ambiguity problem successfully and efficiently. Numerical simulations are given to show the reliability and performance of the proposed method.
\end{abstract}

Index Terms-ambiguity, DOA estimation, MUSIC, unfolded co-prime array.

\section{INTRODUCTION}

D IRECTION of arrival (DOA) estimation has attracted a lot of attention in the last several decades. Numerous DOA estimation algorithms, such as MUSIC [1] and ESPRIT [2], have been proposed for uniform linear arrays (ULA). Unfortunately, due to the restricted distance between adjacent antenna elements, the estimation performance of this antenna geometry is limited by small array aperture and possible mutual coupling effect. Recently, as a promising solution to these drawbacks, DOA estimation with a co-prime linear array has become a research focus [3]. Consisting of two ULAs with inter-element spacing larger than half-wavelength, a co-prime linear array has less mutual coupling effect and an increased array aperture, so a higher resolution and a better estimation performance can be achieved.

Various DOA estimation methods have been proposed for co-prime linear arrays in recent years. A method based on MUSIC is proposed in [4]. The DOAs are obtained by finding the common peaks of the MUSIC spectrums of the two subarrays, and the ambiguities caused by the large inter-element spacing can be eliminated according to the co-prime property. However, this method suffers from the problem of matching error when the number of incoming signals is greater than one. For this problem, an ESPRIT-based method is proposed in [5]. The matching error problem is solved by beamforming based techniques, and without the spectral searching operation, the computational complexity is significantly reduced. Unfortunately, the above mentioned methods treat a co-prime linear

This work is supported by China Scholarship Council (File No.201708440322), and 2017-2019 Sino-French CAI Yuanpei Program.

The authors are with Institut d'Électronique et de Télécommunications de Rennes UMR CNRS 6164, Poltytech de Nantes, Nantes, 44306, France (email: xiao.yang1@etu.univ-nantes.fr). array as two uniform subarrays and perform DOA estimation on them separately. So they have the following problems: (i) the DOF are limited by the subarray with the smallest number of antenna elements; (ii) only the self information of the two subarrays is utilized, which results in a sub-optimal estimation performance; (iii) further processes are required to eliminate the ambiguities caused by the large inter-element spacing.

To tackle these problems, an antenna geometry named unfolded co-prime linear array is proposed in [6]. By unfolding the two subarrays of a general co-prime linear array in two opposite directions, the array aperture can be extended. Furthermore, instead of treating the two subarrays separately, MUSIC is performed with the received signal of the whole array. This technique, having been extended to the scenarios of noncircular signals in [7] and two-dimensional DOA estimation in [8], enjoys the advantages that both self and mutual information can be explored and full DOFs can be obtained. Besides, it has been admitted that the ambiguity problem can be suppressed thanks to the co-prime property. However, it is not always true. When there are two different DOAs having the same directional vectors with the directional vector of a given DOA for the two subarrays respectively, there would still be ambiguous angles of which the corresponding directional vectors can be represented as linear combinations of the directional vectors of true DOAs. As a consequence, the directional vectors of these ambiguous angles are also orthogonal to the noise subspace, which means that some peaks associated with these ambiguous angles will also be found in the MUSIC spectrum. To the best of our knowledge, this problem has never been considered in the open literature.

In this letter, this problem is discussed for the first time, and a modified DOA estimation method is proposed. Firstly, a decision variable is defined to determine the existence of the problem. Then, all the angles associated with the highest spectral peaks, including the true DOAs and the ambiguous angles, are selected out and the Classical Beamforming (CBF) approach is utilized to distinguish them. Simulation results are provided to show the efficiency of the proposed method.

\section{PRoblem Formulation}

Consider an unfolded co-prime array composed of two uniform linear subarrays arranged along two opposite directions, having $M_{1}$ and $M_{2}$ antenna elements, with inter-element spacing $d_{1}=M_{2} \frac{\lambda}{2}$ and $d_{2}=M_{1} \frac{\lambda}{2}$, respectively, where $\lambda$ is the wavelength of incoming signals, and $M_{1}, M_{2}$ are two co-prime integers. Figure 1 shows the case of $M_{1}=7$ and $M_{2}=5$. The element shared by the two subarrays is set as the reference point. The total number of elements is $N=M_{1}+M_{2}-1$.

Assume that there are $K$ ( $K$ is supposed to be known and $K<N$ ) uncorrelated, far-field and narrowband source signals 


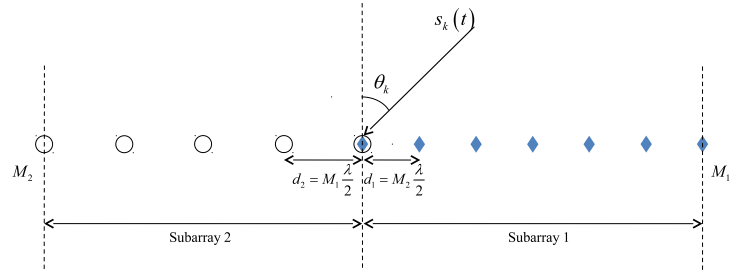

Fig. 1. System Model.

coming from directions $\left\{\theta_{1}, \theta_{2}, \ldots, \theta_{K}\right\}$, with $-90^{\circ}<\theta_{k}<$ $90^{\circ}$ and $1 \leqslant k \leqslant K$, respectively. The received signal can be presented as

$$
\mathbf{x}(t)=\mathbf{A} \mathbf{s}(t)+\mathbf{n}(t)
$$

where $\mathbf{A}=\left[\begin{array}{llll}\mathbf{a}\left(\theta_{1}\right) & \mathbf{a}\left(\theta_{2}\right) & \cdots & \mathbf{a}\left(\theta_{K}\right)\end{array}\right]$ the directional matrix and $\mathbf{a}\left(\theta_{k}\right)=\left[\begin{array}{ll}\mathbf{a}_{1}^{T}\left(\theta_{k}\right) & \mathbf{a}_{2}^{T}\left(\theta_{k}\right)\end{array}\right]^{T}$ the directional vector of the unfolded co-prime array, with $\mathbf{a}_{1}\left(\theta_{k}\right)=$ $\left[\begin{array}{cccc}1 & e^{j M_{2} \pi \sin \theta_{k}} & \cdots & e^{j\left(M_{1}-1\right) M_{2} \pi \sin \theta_{k}}\end{array}\right]^{T}$ and $\mathbf{a}_{2}\left(\theta_{k}\right)=$ $\left[\begin{array}{cccc}e^{-j\left(M_{2}-1\right) M_{1} \pi \sin \theta_{k}} & e^{-j\left(M_{2}-2\right) M_{1} \pi \sin \theta_{k}} & \cdots & e^{-j M_{1} \pi \sin \theta_{k}}\end{array}\right.$ $\mathbf{s}(t)=\left[\begin{array}{llll}s_{1}(t) & s_{2}(t) & \cdots & s_{K}(t)\end{array}\right]^{T}$ denotes the source signal vector with $s_{k}(t)$ the signal transmitted by the $k^{\text {th }}$ source and received at the reference antenna, and $\mathbf{n}(t)$ is the white Gaussian noise vector with zero-mean and covariance matrix $\sigma^{2} \mathbf{I}_{N}$, supposed to be independent from the source signals.

The covariance matrix of the received signal estimated with $L$ snapshots is given by

$$
\widehat{\mathbf{R}}=\frac{1}{L} \sum_{t=1}^{L} \mathbf{x}(t) \mathbf{x}^{H}(t)
$$

and its eigenvalue decomposition can be written as

$$
\widehat{\mathbf{R}}=\mathbf{U}_{s} \boldsymbol{\Lambda}_{s} \mathbf{U}_{s}^{H}+\mathbf{U}_{n} \boldsymbol{\Lambda}_{n} \mathbf{U}_{n}^{H}
$$

where $\mathbf{U}_{s}$ and $\mathbf{U}_{n}$ are composed by the eigenvectors spanning the signal subspace and noise subspace, respectively, and $\boldsymbol{\Lambda}_{s}$ and $\boldsymbol{\Lambda}_{n}$ are the eigenvalue matrices corresponding to $\mathbf{U}_{s}$ and $\mathbf{U}_{n}$, respectively. The pseudo-spectrum of MUSIC can be represented by

$$
P_{M U S I C}(\theta)=\frac{1}{\mathbf{a}^{H}(\theta) \mathbf{U}_{n} \mathbf{U}_{n}^{H} \mathbf{a}(\theta)}
$$

Finally the DOAs can be found by spectral peak-searching of $P_{M U S I C}(\theta)$ over $\left(-90^{\circ}, 90^{\circ}\right)$.

\section{Ambiguity Problem And Proposed Method}

Compared with a conventional co-prime linear array, the unfolded co-prime array can extend the array aperture, obtain full DOFs and avoid the loss of mutual information. Besides, it has been said in [6], [7] that no ambiguities caused by the large inter-element spacing of the subarrays exist in the MUSIC spectrum. However, this is not always true. When there are two different DOAs having the same directional vectors with the directional vector of a given DOA for the two subarrays respectively, there would still be ambiguous angles.

Because of the large inter-element spacing of each subarray, for a given DOA with its associated directional vector, there exist several other angles having the same directional vector for either subarray. Consider that there are three DOAs $\theta_{1}, \theta_{2}$ and $\theta_{3}$, for which the directional vectors satisfy that

$$
\begin{aligned}
& \mathbf{a}_{1}\left(\theta_{1}\right)=\mathbf{a}_{1}\left(\theta_{3}\right) \\
& \mathbf{a}_{2}\left(\theta_{1}\right)=\mathbf{a}_{2}\left(\theta_{2}\right)
\end{aligned}
$$

Then the relationship between $\theta_{1}, \theta_{2}$ and $\theta_{3}$ can be written as

$$
\begin{aligned}
& M_{2} \pi \sin \theta_{1}=M_{2} \pi \sin \theta_{3}+2 k_{1} \pi \\
& M_{1} \pi \sin \theta_{1}=M_{1} \pi \sin \theta_{2}+2 k_{2} \pi
\end{aligned}
$$

with $k_{1}, k_{2}$ two non-zero integers and $k_{1} \in\left(-M_{2}, M_{2}\right), k_{2} \in$ $\left(-M_{1}, M_{1}\right)$.

From Equations (7) and (8), it can be deduced that

$$
\sin \theta_{2}-\sin \theta_{3}=\frac{2}{M_{2}} k_{1}-\frac{2}{M_{1}} k_{2}
$$

which can be re-written as

$$
\sin \theta_{2}+\frac{2}{M_{2}}\left(-k_{1}\right)=\sin \theta_{3}+\frac{2}{M_{1}}\left(-k_{2}\right)
$$

Defining the term $\sin \theta_{4}$ and expressing Equation (10) in two following equations as

$$
\begin{aligned}
& \sin \theta_{4}=\sin \theta_{2}+\frac{2}{M_{2}}\left(-k_{1}\right) \\
& \sin \theta_{4}=\sin \theta_{3}+\frac{2}{M_{1}}\left(-k_{2}\right)
\end{aligned}
$$

then it comes that

$$
\begin{aligned}
& M_{2} \pi \sin \theta_{4}=M_{2} \pi \sin \theta_{2}+2\left(-k_{1}\right) \pi \\
& M_{1} \pi \sin \theta_{4}=M_{1} \pi \sin \theta_{3}+2\left(-k_{2}\right) \pi
\end{aligned}
$$

Then the follwing equations are obtained

$$
\begin{aligned}
& \mathbf{a}_{1}\left(\theta_{4}\right)=\mathbf{a}_{1}\left(\theta_{2}\right) \\
& \mathbf{a}_{2}\left(\theta_{4}\right)=\mathbf{a}_{2}\left(\theta_{3}\right)
\end{aligned}
$$

According to Equations (5), (6) and Equations (15), (16), there is the relationship between the directional vectors of $\theta_{1}$, $\theta_{2}, \theta_{3}$ and $\theta_{4}$ as

$\left[\begin{array}{l}\mathbf{a}_{1}\left(\theta_{1}\right) \\ \mathbf{a}_{2}\left(\theta_{1}\right)\end{array}\right]-\left[\begin{array}{l}\mathbf{a}_{1}\left(\theta_{2}\right) \\ \mathbf{a}_{2}\left(\theta_{2}\right)\end{array}\right]-\left[\begin{array}{l}\mathbf{a}_{1}\left(\theta_{3}\right) \\ \mathbf{a}_{2}\left(\theta_{3}\right)\end{array}\right]+\left[\begin{array}{l}\mathbf{a}_{1}\left(\theta_{4}\right) \\ \mathbf{a}_{2}\left(\theta_{4}\right)\end{array}\right]=0$

It can be seen that the directional vector of $\theta_{4}$ can be represented as a linear combination of the directional vectors of the other three angles, and when there are three signals coming from any three of the four angles, the directional vector of the remaining one would lie in the signal subspace, and be also orthogonal to the noise subspace, resulting in an ambiguous peak in the MUSIC spectrum. Generally, for an arbitrary number of incoming signals (at least three), if the DOAs of three of them meet the conditions given by Equations (5) and (6), then the ambiguity problem occurs.

Consider that $M_{1}=7, M_{2}=5$, and three signals come from $\theta_{1}=10.00^{\circ}, \theta_{2}=27.35^{\circ}$ and $\theta_{3}=35.01^{\circ}$, respectively, satisfying Equations (5) and (6). The simulation result of the method proposed in [6] is shown in Figure 2, in which the signal-to-noise ratio (SNR) is $10 \mathrm{~dB}$ and the number of snapshots is 200 . It is clear that apart from the three true peaks, 


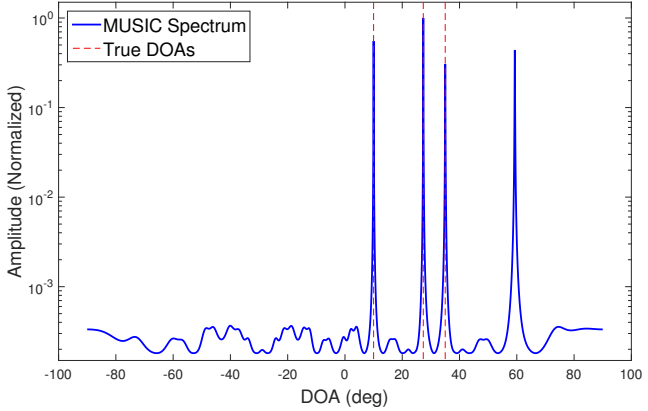

Fig. 2. Ambiguity Problem.

there is an ambiguous peak at $\theta_{4}=59.25^{\circ}$, satisfying Equations (15) - (17). For this antenna array configuration, the ambiguity problem also occurs in the cases of $\left\{\theta_{1}, \theta_{2}, \theta_{3}\right\}=$ $\left\{20.00^{\circ}, 38.88^{\circ}, 47.90^{\circ}\right\},\left\{30.00^{\circ}, 12.37^{\circ}, 64.16^{\circ}\right\}$ and others satisfying Equations (5) and (6), which is a real problem in this method.

In order to solve the ambiguity problem, we propose a modified method. Firstly, it is required to determine if the problem exists or not. The MUSIC spectrum is expected to exhibit $K$ high peaks associated with the $K$ true DOAs. However, when some ambiguous peaks exist, more than $K$ high peaks will be found. Suppose that there are totally $Q$ peaks in the MUSIC spectrum, of which the locations and amplitudes are denoted as $\left(\widehat{\theta}_{p}, y_{p}\right),(p=1,2, \cdots, Q)$. Sorting $y_{p}$ in descending order, we have

$$
y_{1}>y_{2}>\cdots>y_{Q}
$$

Define a decision variable as

$$
D(n)=\frac{y_{n}-y_{n+1}}{y_{n+1}},(n=1,2, \cdots, Q-1)
$$

It can be noticed that when both $y_{n}$ and $y_{n+1}$ are two amplitudes of high peaks or low peaks, the decision variable $D(n)$ is small. If $y_{n}$ is the amplitude of a high peak and $y_{n+1}$ is the amplitude of a low peak, the decision variable $D(n)$ becomes much larger. Thus the number of high peaks can be decided by

$$
J=\arg \max _{n} D(n)
$$

When $J>K$, the ambiguity problem is considered as existing.

To distinguish the true DOAs and the ambiguous angles, the Classical Beamforming (CBF) approach can be utilized. The $J$ angles associated with the $J$ highest peaks $\widehat{\theta}_{q},(q=$ $1,2, \cdots, J)$ are selected out, then the $J$ candidate directional vectors can be constructed with these candidate DOAs as $\mathbf{a}\left(\widehat{\theta}_{q}\right)=\left[\begin{array}{ll}\mathbf{a}_{1}^{T}\left(\widehat{\theta}_{q}\right) & \mathbf{a}_{2}^{T}\left(\widehat{\theta}_{q}\right)\end{array}\right]^{T}$. The CBF powers of the $J$ candidate directional vectors can be calculated by

$$
P_{C B F, q}=\frac{\mathbf{a}^{H}\left(\widehat{\theta}_{q}\right) \widehat{\mathbf{R}} \mathbf{a}\left(\widehat{\theta}_{q}\right)}{N^{2}}
$$

Signals come from true DOAs and there is no physical signal coming from ambiguous angles. So the CBF power at the true DOAs is greater than that at the ambiguous angles. By finding the $K$ greatest results in Equation (21), the true DOAs can be obtained.
TABLE I

DECISION VARIABLE

\begin{tabular}{c|cccccc}
\hline$n$ & 1 & 2 & 3 & 4 & 5 & $6-29$ \\
\hline$D(n)$ & 0.8098 & 0.2572 & 0.4372 & 833.58 & 0.0046 & $<0.3$ \\
\hline
\end{tabular}

TABLE II

CBF POWERS AND TEST RESUlT

\begin{tabular}{c|cccc}
\hline$\widehat{\theta}_{q}$ & $10.00^{\circ}$ & $35.02^{\circ}$ & $27.36^{\circ}$ & $59.31^{\circ}$ \\
\hline$P_{C B F, q}$ & 1.4465 & 1.2200 & 1.1740 & 0.4997 \\
\hline True or Ambiguous & True & True & True & Ambiguous
\end{tabular}

Compared with the existing ambiguity-solving methods in [9] and [10], in which the ambiguities are suppressed by rotating the antenna array physically [9] and the true DOAs are discriminated from the ambiguous ones by estimating the signal power values with a high-dimensional cost function by DFP and BFGS algorithms [10], the proposed method has a lower computational complexity without any specific requirement for the antenna array. Therefore, it is more suitable for real applications.

\section{Simulations AND ANAlysis}

\section{A. Estimation Performance}

To illustrate the principle of the proposed method, consider the ambiguity problem case of which the simulation result is shown in Figure 2. Select out the locations and amplitudes of all the peaks in the MUSIC spectrum and calculate the decision variable $D(n)$ (19). The obtained results are shown in Table I. According to the maximum of $D(n)$, there are $J=4$ peaks obviously higher than the others, corresponding to three true DOAs and one ambiguous angle. Select out the $J=4$ candidate angles and calculate their corresponding CBF powers $P_{C B F, q}(21)$. The obtained results are shown in Table II. It is clear that the $\mathrm{CBF}$ powers at the three true DOAs are greater than that at the ambiguous angle. So the true DOAs can be distinguished among the candidate angles, and the ambiguity problem is solved successfully.

The ambiguity problem does not affect the estimation accuracy, but invalidates the estimation by introducing nonexisting DOAs. The proposed method can achieve the same estimation performance as the existing method when there is no ambiguity problem, and keep robust and reliable when the problem occurs. To reveal the superiority of the proposed method in ambiguity problem cases, the reliability comparison of the proposed method and the method proposed in [6] is shown in Figure 3, with 20 independent simulations, in which SNR is $10 \mathrm{~dB}$ and the number of snapshots is 200. The three estimated angles with the highest peak amplitudes and the greatest $\mathrm{CBF}$ powers are chosen as the final estimation results for the method in [6] and the proposed method, respectively. It can be seen that the estimation results of the method in [6] are ambiguous and the unsolved ambiguity problem reduces the rate of success of DOA estimation. In contrast, the proposed method can solve the ambiguity problem successfully, and the performance remains stable and remarkable. 

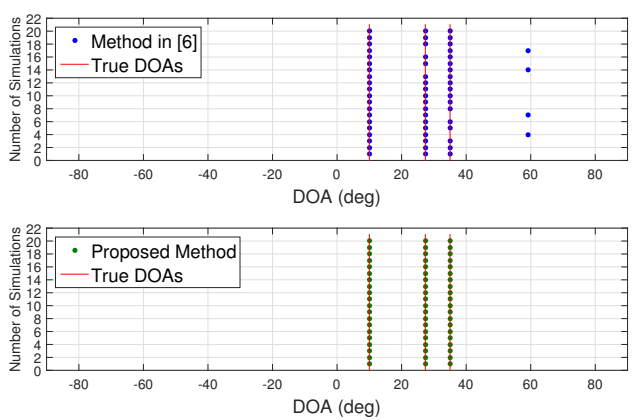

Fig. 3. Reliability Comparison.

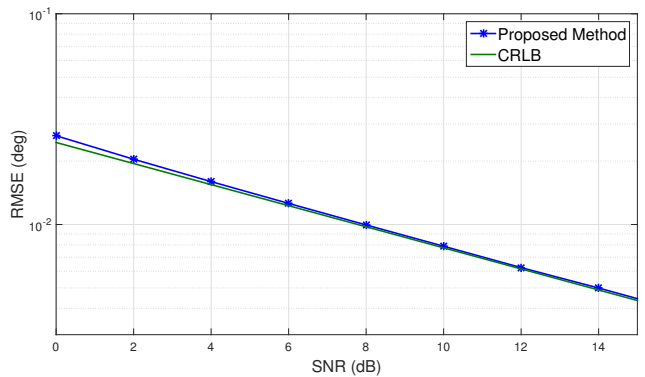

Fig. 4. RMSE Performance versus SNR.

To assess the DOA estimation performance of the proposed method, the Root Mean Square Error (RMSE) criteria is used as performance measurement, which is defined as

$$
\operatorname{RMSE}=\sqrt{\frac{1}{K G} \sum_{k=1}^{K} \sum_{g=1}^{G}\left(\widehat{\theta}_{g, k}-\theta_{k}\right)^{2}}
$$

where $K$ is the number of incoming signals, $G$ the number of Monte Carlo trials, and $\widehat{\theta}_{g, k}$ the estimate of the true DOA $\theta_{k}$ of $g^{\text {th }}$ Monte Carlo trial. $G=500$ is used. An unfolded co-prime linear array with $M_{1}=7$ and $M_{2}=5$ is considered. The Cramér-Rao Lower Bound (CRLB) for this array geometry is also given as a benchmark [11].

Figure 4 and Figure 5 depict the RMSE performance of the proposed method versus SNR $(L=200)$ and the snapshots number $(\mathrm{SNR}=10 \mathrm{~dB})$, respectively, with $K=3$ signals coming from $\theta_{1}=10.00^{\circ}, \theta_{2}=27.35^{\circ}$ and $\theta_{3}=35.01^{\circ}$. It can be seen that in spite of the existence of the ambiguity problem, the performance of the proposed method remains remarkable and close to the CRLB. So the proposed method has a wider range of applications than the original method.

\section{B. Complexity Analysis}

According to the principle of the proposed method, the computational complexity will be different, depending on the existence of the ambiguity problem. When the problem does not exist, the complexity of the proposed method is $O\left(N^{2} L+N^{3}+T N(N-K)\right)$, where $T$ is the number of spectral peak-searching, which is the same as the method proposed in [6]. When the problem exists, the complexity of the proposed method is $O\left(N^{2} L+N^{3}+T N(N-K)+J N^{2}\right)$, which slightly exceeds the method in [6].

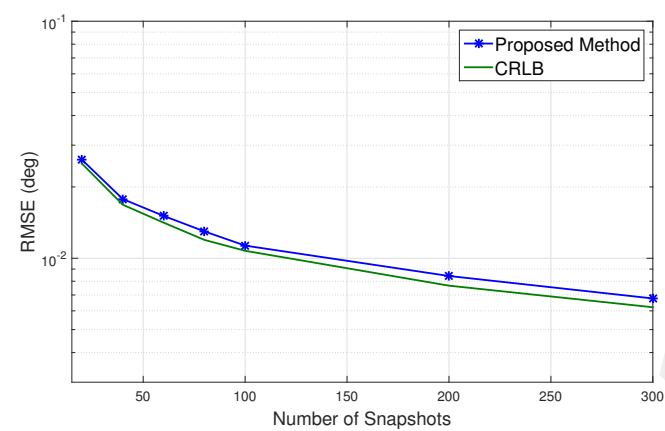

Fig. 5. RMSE Performance versus Snapshots Number.

\section{CONCLUSION}

In this letter, DOA estimation with an unfolded co-prime array is investigated, and the ambiguity problem is discussed for the first time. We show that there would be ambiguous peaks in the MUSIC spectrum when there are two different DOAs having the same directional vectors with the directional vector of a given DOA for the two subarrays respectively. In addition, a decision variable is defined to determine the existence of the problem, and then a modified method based on the Classical Beamforming technique is proposed to distinguish the true DOAs. Simulation results show that the proposed method can solve the ambiguity problem successfully with a slight additional complexity burden. So it can achieve a wider range of applications. As a future work, it is worth extending the study of the ambiguity problem generated by more than three sources.

\section{REFERENCES}

[1] R. Schmidt, "Multiple emitter location and signal parameter estimation," IEEE transactions on antennas and propagation, vol. 34 , no. 3, pp. 276 $280,1986$.

[2] R. Roy and T. Kailath, "ESPRIT-estimation of signal parameters via rotational invariance techniques," IEEE Transactions on acoustics, speech, and signal processing, vol. 37, no. 7, pp. 984-995, 1989.

[3] P. P. Vaidyanathan and P. Pal, "Sparse sensing with co-prime samplers and arrays," IEEE Transactions on Signal Processing, vol. 59, no. 2, pp. 573-586, 2011.

[4] C. Zhou, Z. Shi, Y. Gu, and X. Shen, "DECOM: DOA estimation with combined MUSIC for coprime array," in Wireless Communications \& Signal Processing (WCSP), 2013 International Conference on. IEEE, 2013, pp. 1-5.

[5] F. Sun, B. Gao, L. Chen, and P. Lan, "A low-complexity ESPRIT-based DOA estimation method for co-prime linear arrays," Sensors, vol. 16 no. 9 , p. $1367,2016$.

[6] W. Zheng, X. Zhang, P. Gong, and H. Zhai, "DOA estimation for coprime linear arrays: An ambiguity-free method involving full DOFs." IEEE Communications Letters, vol. 22, no. 3, pp. 562-565, 2018.

[7] H. Zhai, X. Zhang, W. Zheng, and P. Gong, "DOA estimation of noncircular signals for unfolded coprime linear array: Identifiability, and algorithm (may 2018)," IEEE Access, 2018.

[8] P. Gong, X. Zhang, and W. Zheng, "Unfolded coprime L-shaped arrays for two-dimensional direction of arrival estimation," International Journal of Electronics, vol. 105, no. 9, pp. 1501-1519, 2018.

[9] M. Lin, P. Liu, and J. Liu, "DOA ambiguity suppressing by rotating the array," in Antennas and Propagation (APCAP), 2015 IEEE 4th AsiaPacific Conference on. IEEE, 2015, pp. 165-167.

[10] Z. He, Z. Zhao, Z. Nie, P. Tang, J. Wang, and Q.-H. Liu, "Method of solving ambiguity for sparse array via power estimation based on MUSIC algorithm," Signal Processing, vol. 92, no. 2, pp. 542-546, 2012.

[11] P. Stoica and A. Nehorai, "Performance study of conditional and unconditional direction-of-arrival estimation," IEEE Transactions on Acoustics, Speech, and Signal Processing, vol. 38, no. 10, pp. 17831795, 1990. 